\title{
Relationship between type II diabetes mellitus and Helicobacter pylori infection in Erbil city
}

\begin{tabular}{|c|c|c|c|c|}
\hline Rawaz D. Tawfeeq* & Zahra A. Amin** & Sheila M. Nuraddin** & Aveen J alal*** & Sayran K. Hama Baiz* \\
\hline
\end{tabular}

Background and objective: Type 2 diabetes mellitus is a metabolic disorder characterized by the increase in blood glucose due to insulin resistance or deficiency of insulin. The subjects are more likely to be prone to infection. So, it could be correlated with Helicobacter pylori infection, which means that gastrointestinal inflammation might be affected by uncontrolled glycemic level. This study aimed to examine the correlation of type II diabetes and infection of gastrointestinal in order to illustrate such complication of diabetes mellitus apart from others.

Methods: A total of 64 persons from Erbil city participated in this cross-sectional study. They were divided into two groups, each group involving 32 persons. The cases group included those suffering from type II diabetes and were selected by simple random sampling method. The other group included those not possessing any types of disease including diabetes mellitus. Examination of Helicobacter pylori, glycated hemoglobin (HbA1c), besides measuring blood pressure and body mass index were performed for all individual subjects in both groups. Chi-Square and unpaired t-test were used for data analysis.

Results: There was a 59\% positively Helicobacter pylori in diabetes group whereas there was a $31 \%$ positively Helicobacter pylori in non-diabetes mellitus. The difference between the rates of Helicobacter pylori in both groups was statistically significant $(P<0.05)$.

Conclusion: The prevalence of Helicobacter pylori infection in diabetics was significantly higher than the non-diabetics.

Keywords: Type II diabetes mellitus; H. pylori infection; HbA1c.

\section{Introduction}

Type 2 Diabetes mellitus (T2DM) could be part of the metabolic syndrome or could be a single metabolic disorder, emerging pandemic; responsible for an estimated 3.8 million adult deaths worldwide. ${ }^{1}$ It is also the fourth major cause of mortality worldwide. The complex metabolic disease mostly results from the interaction between genetic and environmental risk factors. Environmental risk factors for T2DM include obesity, sedentary lifestyle, smoking, high fat/ cholesterol diet, refined carbohydrates, and some psychological factors. This metabolic disease requires effective long-term management to achieve optimal glycemic control and minimize chronic complications. ${ }^{2}$ Symptoms of high blood sugar include frequent urination, increased thirst, and increased hunger. If diabetes mellitus (DM) is left untreated, it can cause many complications. Acute complications can include diabetic ketoacidosis, non-ketotic hyperosmolar coma, or death. Serious long-term complications include heart disease, stroke, chronic kidney failure, foot ulcers, and damage to the eyes. ${ }^{3}$ Helicobacter pylori $(\mathrm{H}$. pylori) were previously named Campylobacter pylori, which are a Gramnegative, microaerophilic bacterium found in the stomach and sometimes can be

* Department of Clinical Analysis, College of Pharmacy, Hawler Medical University, Erbil, I raq.

** Department of Pharmacognosy, College of Pharmacy, Hawler Medical University, Erbil, I raq.

$* * *$ Ministry of Health, Erbil, I raq. 
present in other parts of the body. It was identified and linked to peptic ulcers in 1982 by Australian scientists Barry Marshall and Robin Warren for which in 2005 they were awarded the Nobel Prize in Physiology or Medicine. ${ }^{4} \mathrm{H}$. Pylori is one of the most common chronic bacterial infections in humans. It occurs throughout the world and causes several gastro duodenal diseases including gastric ulcer, duodenal ulcer, gastric MALT lymphoma, and distal gastric cancer. $\mathrm{H}$. Pylori strains may be divided into at least two subgroups based on the expression (type I) or nonexpression (type II) of cytotoxin-associated gene $\mathrm{A}(\mathrm{CagA})$ and the vacuolating cytotoxin. The CagA has been identified as a major virulence factor of $\mathrm{H}$. pylori. It has been reported that the CagA-positive $\mathrm{H}$. pylori strains induce more severe gastric mucosal inflammation than CagA-negative strains. The CagApositive $\mathrm{H}$. pylori strains are associated with higher risks of peptic ulcer disease and gastric cancer. In addition to the gastrointestinal symptoms, some extra-gastric diseases such as cardiovascular diseases, lung diseases, hematologic diseases, eye and skin diseases, hepatobiliary diseases, and neurological disorders have been also linked to $\mathrm{H}$. pylori infection. It has been reported that $\mathrm{H}$. pylori infection may also be responsible for some endocrine disorders, such as autoimmune thyroid diseases, diabetes mellitus, and primary hyperparathyroidism. ${ }^{5}$ The relationship between $\mathrm{H}$. pylori and diabetes mellitus was first explored in 1989 by Simon et al., ${ }^{6}$ who found that the prevalence of $\mathrm{H}$. pylori infection in patients with diabetes mellitus was significantly higher than in asymptomatic controls (62\% vs. 21\%). However, the test used for detecting $\mathrm{H}$. pylori was only a rapid urease test, and their comparison did not adjust for age, which is a major confounding factor. Diabetic patients are more susceptible to infection than others by different mechanisms. Firstly, a diabetes-induced impairment of cellular and humoral immunity may enhance an individual's sensitivity to $\mathrm{H}$. pyloriinfection. ${ }^{7}$ Secondly, diabetes-induced reduction of gastrointestinal motility and acid secretion may promote pathogen colonization and infection rate in the gut. ${ }^{8}$ Thirdly, altered glucose metabolism may produce chemical changes in the gastric mucosa that promote $\mathrm{H}$. pyloricolonization. ${ }^{9}$ Finally, individuals with diabetes are more frequently exposed to pathogens than their healthy counterparts as they regularly attend hospital settings. ${ }^{10}$ This cross-sectional study aimed to examine the association between type II diabetes mellitus and Helicobacter pylori infection in order to distinguish the influence of type II DM on such infection. The $\mathrm{HbA} 1 \mathrm{c}$ and $\mathrm{H}$. pylori infection parameters are measured in subjects with DM group, and then they are compared to its counterpart group.

\section{Methods}

A total of 64 subjects of Kurdish nationality participated in this study; 32 of them were non-diabetic volunteers, and 32 of participants were suffering from type II diabetes mellitus at Layla Qasm health center, which is the only center for DM disease in Erbil. First of all, the permission was taken from all of the subjects by written consent form. Then, their height and weight were measured and followed by calculating BMI weight/height ${ }^{2}$. Then, their ages were taken in the current study as shown. The exclusion criteria included the patients who were already suffering $\mathrm{H}$. Pylori infection, and they were on treatment for this disease before choosing as subjects. Besides, those were on regular long-term non-steroidal antiinflammatory drugs treatment and taking a regular interval of proton pump inhibitor or $\mathrm{H} 2$ blocker. On the other hand, inclusion criteria involved those who suffered from T2DM for more than three years in the diabetic group whereas volunteers in the control group should be non-diabetic and their HbA1c less than $6.4 \%$. All patients 
in the DM group were taken with the permission of the physician specialist at the health center. Subjects were randomly selected (simple random selected method) for this study at the diabetes health center in Erbil. The whole study was carried out from October 2016 to April of 2017 and this duration, 64 volunteers taken subjected to test $\mathrm{HbA1c}$ and $\mathrm{H}$. pylori apart from anthropometric measurements. First of all, permission from all subjects was taken. Then they were asked to come in the morning and fasting for at least 12 hours. Secondly, the blood sample was taken from all of them. Then, all samples were placed into the first tube containing sodium citrate as the anticoagulant, the second tube containing heparin as anticoagulant then it was shaken to prevent hemolysis. It was followed by the centrifugation of the first tube, and the sample was placed in a machine called autoanalyzer(COBAS) in order to measure HbA1c. For detecting H.pylori, the sample was taken from sodium citrate tube after that some drop of the sample was placed on kit manufactured for this test and five should be waited. Finally, the result of $\mathrm{H}$. pylori had to be recorded. A cross-sectional study with a comparison group was designed for this research, which includes the rate of $\mathrm{H}$. pylori in the DM group while the rate of $\mathrm{H}$. pylori in the non-DM group. All volunteers were first requested to measure weight, height, blood pressure and secondly $\mathrm{HbA1C}$ with fasting blood sugar in the morning, and finally asked to assess $\mathrm{H}$. Pylori infection. All subjects in diabetic groups were diagnosed as non-insulin DM by physicians at Layla Qasm hospital. In terms of statistical analysis in this study, the Chi-square test was used to find out the association between $\mathrm{HbA1c}$ and $\mathrm{H}$. pylori, between gender and $\mathrm{H}$, pylori, and between $\mathrm{BMI}$ and $\mathrm{H}$. pylori. Chi-square was used to determine statistical differences between the numbers of positivity's $\mathrm{H}$. pylori between diabetic and non-diabetics groups. To compare differences in genders between these two groups in the current study, Chi-Square was utilized. Besides, unpaired t-test (independent t-test) was used to determine the differences of all means between these two groups in this study. All data in this study was displayed as average \pm SEM and the $P$ value $<0.05$ was used to indicate statistical significance. Stata version 12 was utilized to perform statistical comparisons and analysis in this study.

\section{Results}

All the 64 volunteers with or without DM successfully completed all requirement measurements such as HbA1c and $\mathrm{H}$. pylori investigation. The same number of subjects in both non-diabetic and diabetic groups was participated, involving 32 for each group. This study included 11 males and 21 females in the diabetic group while 19 males and 13 females in the non-diabetic group. In both groups. They had similar age, height, weight, and BMI since their $P$ values were not significantly different as shown in Table 1.

Table 1: Anthropometric parameters of subjects.

\begin{tabular}{lccc}
\hline & Diabetic & Non-diabetic & $P$ value \\
\hline Number of subjects & 32 & 32 & \\
Gender & 11 Male, 21 female & 19 Male, 13 female & \\
Age, years & $55 \pm 1.5$ & $51.4 \pm 0.9$ & 0.057 \\
Height, cm & $158.4 \pm 1.2$ & $161.3 \pm 1.4$ & 0.338 \\
Weight, kg & $71.1 \pm 2.9$ & $75.5 \pm 2.1$ & 0.060 \\
BMI, kg/m2 & $28.5 \pm 0.7$ & $29.2 \pm 0.7$ & 0.495 \\
\hline Values are Means \pm SEM. Abbreviations, BMl: body mass index. & \\
\hline
\end{tabular}




\section{Age and gender admeasurement}

There were not any significant differences in gender regardless of DM and non-DM group. Even though the numbers of the males in the non-DM group were higher than that of the females in the same group while the number of females in DM group was higher than that of the males in the same group. There was no any statistical significance in gender. In age division, including 40-50 years, 51-60 years and, more than 60 years old, the difference among these ages did not demonstrate any statistically significant. In age 40-50, the numbers of subjects were 12 and 15 in diabetic and non-diabetics groups, respectively. At age 51-60, 11 participants were in the DM group, and 15 are in the non-DM group. In the age of more than 60 , the number of subjects was nine and two in DM and non-DM respectively, as illustrated in Table 2.

\section{BMI parameters}

The ratios of obese, overweight and normal populations in DM group were the same to those in the non-DM group, which means that there were no any statistically significant differences in such ratios between the two groups. Numbers of participants of normal, overweight and obese in DM group were seven, 14 and 11 , respectively. Besides, the number of participants of normal, overweight and obese in the non-DM group were three, 17 and 12 respectively, as shown in Table 3.The distinguishing obese, overweight and normal weight were performed based on BMI measurements.

Table 2: Distribution of the age and gender of the two groups.

\begin{tabular}{llcccccc}
\hline & \multicolumn{5}{c}{ Number of H. pylori (\%) with differentiation age and gender } \\
& Age & Male & Female & Male & Female & No. & $\%$ \\
\hline Diabetics & $40-50$ & $3(25 \%)$ & $6(50 \%)$ & $2(16.7 \%)$ & $1(8.3 \%)$ & 12 & 100 \\
& $51-60$ & $2(18.2 \%)$ & $3(27.3 \%)$ & $1(9.1 \%)$ & $5(45.4 \%)$ & 11 & 100 \\
& $>60$ & $2(22.2 \%)$ & $3(33.3 \%)$ & $1(11.2 \%)$ & $3(33.3 \%)$ & 9 & 100 \\
\multirow{2}{*}{ Non-diabetics } & $40-50$ & $4(26.6 \%)$ & $1(6.8 \%)$ & $6(40 \%)$ & $4(26.6 \%)$ & 15 & 100 \\
& $51-60$ & $0(0.0 \%)$ & $5(33.4 \%)$ & $6(40 \%)$ & $4(26.6 \%)$ & 15 & 100 \\
& $>60$ & $0(0.0 \%)$ & $0(0.0 \%)$ & $1(50 \%)$ & $1(50 \%)$ & 2 & 100 \\
\multirow{2}{*}{ Total } & & $11(17.3 \%)$ & $18(28.1 \%)$ & $17(26.5 \%)$ & $18(28.1 \%)$ & 64 & 100 \\
\hline
\end{tabular}

Table 3: Separation of the weight of subjects with BMI measurements in both groups.

\begin{tabular}{llccccccc}
\hline & & \multicolumn{3}{c}{ Prevalence of H. pylori by BMI } & \multicolumn{2}{c}{ Total } & \multirow{2}{*}{ P value } \\
& BMI & No. & $\%$ & No. & $\%$ & No. & $\%$ & \\
\hline Diabetics & Normal & 5 & 71.4 & 2 & 28.6 & 7 & 100 & 0.600 \\
& Overweight & 7 & 50 & 7 & 50 & 14 & 100 & \\
& Obese & 7 & 63.6 & 4 & 36.4 & 11 & 100 & \\
\multirow{5}{*}{ Non-diabetics } & Normal & 0 & 0 & 3 & 100 & 3 & 100 & 0.079 \\
& Overweight & 4 & 23.5 & 13 & 76.5 & 17 & 100 & \\
& Obese & 6 & 50 & 6 & 50 & 12 & 100 & \\
Total & 29 & & 35 & & 64 & 100 & \\
\hline \hline
\end{tabular}


Prevalence of $\mathbf{H}$. pylori infection suffering from $\mathrm{H}$. pylori, and $33.3 \%$ of correlated to HbA1c

As demonstrated in Table 4, there were no any patients with DM whose HbA1c was less than $5.5 \%$ while $\mathrm{HbA} 1 \mathrm{c}$ in the majority of volunteers without DM was less than $5.5 \%$, involving 24 participants. Around $75 \%$ of volunteers without DM did not suffer from $\mathrm{H}$. pylori in the group whose $\mathrm{HbA} 1 \mathrm{c}$ was less than $5.5 \%$. There were no any subjects without DM in control group with more $6.5 \% \mathrm{HbA} 1 \mathrm{c}$. In $6.5 \% \mathrm{HbA1c}$ group, subjects with positive $\mathrm{H}$. pylori were more than that of with negative $\mathrm{H}$. pylori (58.6\% against $41.4 \%$ ). In $5.5 \%$ to $6.5 \%$ HbA1c group, there were negative and positive $\mathrm{H}$. pylori in both case and control group. In DM group, $66.7 \%$ of subjects subjects did not suffer from such infection whereas in non-DM there were 50\% in both negative and positive with $\mathrm{H}$. pylori in $5.5-6.5 \% \mathrm{HbA} 1 \mathrm{c}$. There was a significant difference in HbA1c in both groups $(P=0.04)$. This means that increased the rate of $\mathrm{H}$. pylori were directly proportional to augmenting $\mathrm{HbA} 1 \mathrm{c}$ measurements.

$\mathrm{H}$. Pylori infection in both diabetic and non-diabetic group

In Figure 1, there was a significant difference in $\mathrm{H}$. pylori between both groups, diabetic and non-diabetic group. The parameter of $\mathrm{H}$. pylori infection in a diabetic patients was much higher than the non-diabetic group $(P=0.024)$.

Table 4: Prevalence of $\mathrm{H}$. pylori infection in the different range of $\mathrm{HbA1C}$.

\begin{tabular}{lcccccccc}
\hline & \multicolumn{9}{c}{ Prevalence of H.pylori infection } & \multicolumn{2}{c}{ Total } & \\
& HbA1c & No. & $\%$ & No. & $\%$ & No. & $\%$ & $P$ value \\
\hline Diabetics & $<5.5$ & 0 & 0 & 0 & 0 & 0 & 100 & \\
& $5.5-6.5$ & 2 & 66.7 & 1 & 33.3 & 3 & 100 & \\
& $>6.5$ & 17 & 58.6 & 12 & 41.4 & 29 & 100 & \\
\multirow{4}{*}{ Non-diabetics } & $<5.5$ & 6 & 25 & 18 & 75 & 24 & 100 & \\
& $5.5-6.5$ & 4 & 50 & 4 & 50 & 8 & 100 & \\
\multirow{4}{*}{ Total } & $>6.5$ & 0 & 0 & 0 & 0 & 0 & 100 & \\
& & 29 & 45.3 & 35 & 54.7 & 64 & 100 & 0.04 \\
\hline
\end{tabular}
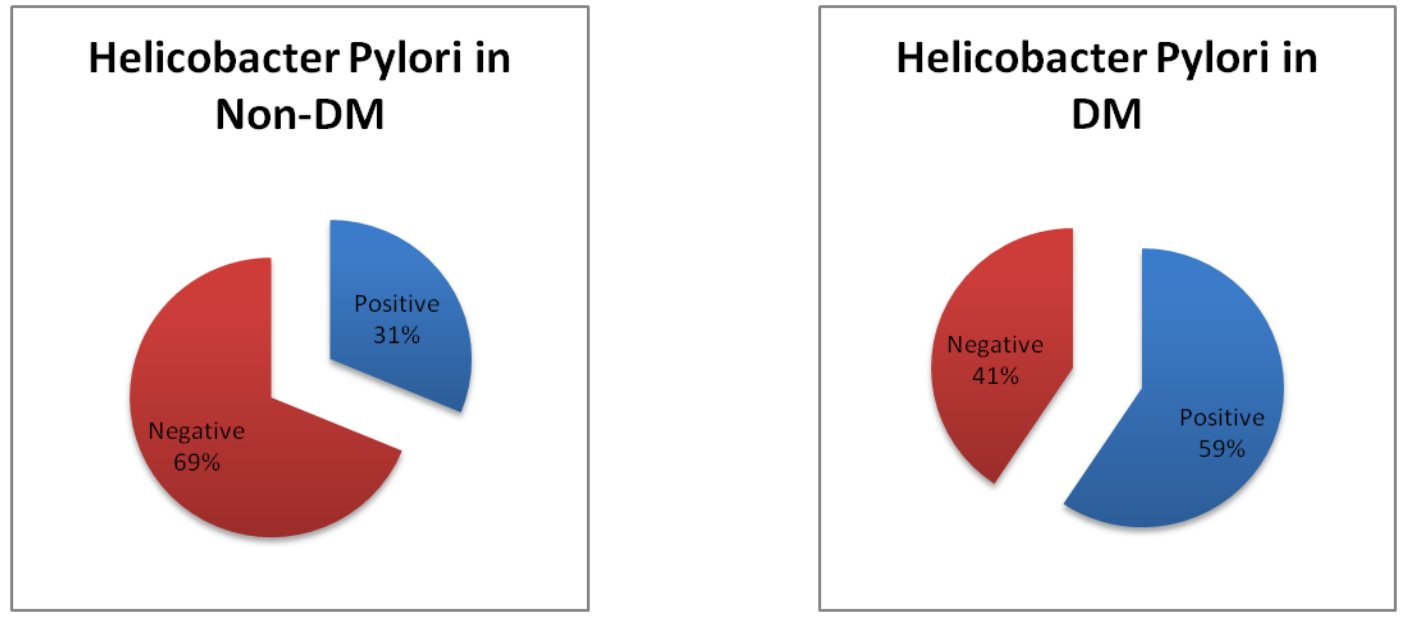

Figure 1: Ratio of H. pylori infection in both diabetic and non-diabetic groups. 
HbA1c measurement in diabetic and non-diabetic groups

The percent of $\mathrm{HbA} 1 \mathrm{c}$ in the DM group was significantly higher compared to that of the non-DM group $(P=0.02)$ as illustrated in Figure 2.

\section{Discussion}

In many studies, the prevalence of $\mathrm{H}$. pylori infection was significantly higher in type 2 diabetic mellitus than in non-diabetic mellitus had been discussed. ${ }^{11-13}$ First of all, the aim of our investigation was to study the correlation between type two diabetic mellitus and $\mathrm{H}$. pylori infection in our community. This means that it was demonstrated the effect of increasing $\mathrm{HbA1c}$ on $\mathrm{H}$. pylori infection in the population. So therefore for this purpose, we perform a test for $\mathrm{HbA} 1 \mathrm{c}$ and $\mathrm{H}$. pylori for each subject. The main fining in the current study was that type II DM affects the possessing the $\mathrm{H}$. pylori infection. During this study, sixty-four volunteer was included, and all of them were taken, they should not be on regular interval any of this medication like antibiotic or $\mathrm{PPI}, \mathrm{H}_{2}$ blocker since if a patient takes this medication may Cause false negative result of $\mathrm{H}$. pylori infection. The $\mathrm{H}$. pylori infection prevalence is higher in-patient with high-level $\mathrm{HbA1c}$ while the patient with a low level of HbA1c; the ratio of suffering $\mathrm{H}$. pylori is lower level.
Furthermore, the patient with a high level of $\mathrm{HbA} 1 \mathrm{c}$ and $\mathrm{H}$. pylori infection are mostly with high BMI rather than low BMI. High levels of BMI indicate that the patient had high blood glucose and fat deposition (dyslipidemia) and more prone to inflammation. This means that they affect insulin secretion and insulin receptor resistance by reducing gastric motility cause colonization of mycobacteria in stomach cause complications and lowgrade inflammation of lining mucosa and ulcerations. T2DM is more common in patients more than 40 years old, particularly in the obese population. In this study, $59 \%$ of patients with T2DM have $\mathrm{H}$. pylori infection while only $31 \%$ of volunteers in the control group posses $\mathrm{H}$. pylori. This means that $\mathrm{H}$. pylori in patients with T2DM are significantly higher than that in these without T2DM, p-value equals to 0.024 . The reason behind $31 \%$ of the control population with $\mathrm{H}$. pylori in this study is that they are overweight and close to obese rather than healthy weight. There is no significant difference in BMI between diabetic and non-diabetic groups in the present study. In general, the prevalence of $\mathrm{H}$. pylori could nowadays be high in our community due to lifestyle risk including living in crowding home and eating of junk food, the unsanitary source of water supply, family member infection, high BMI.

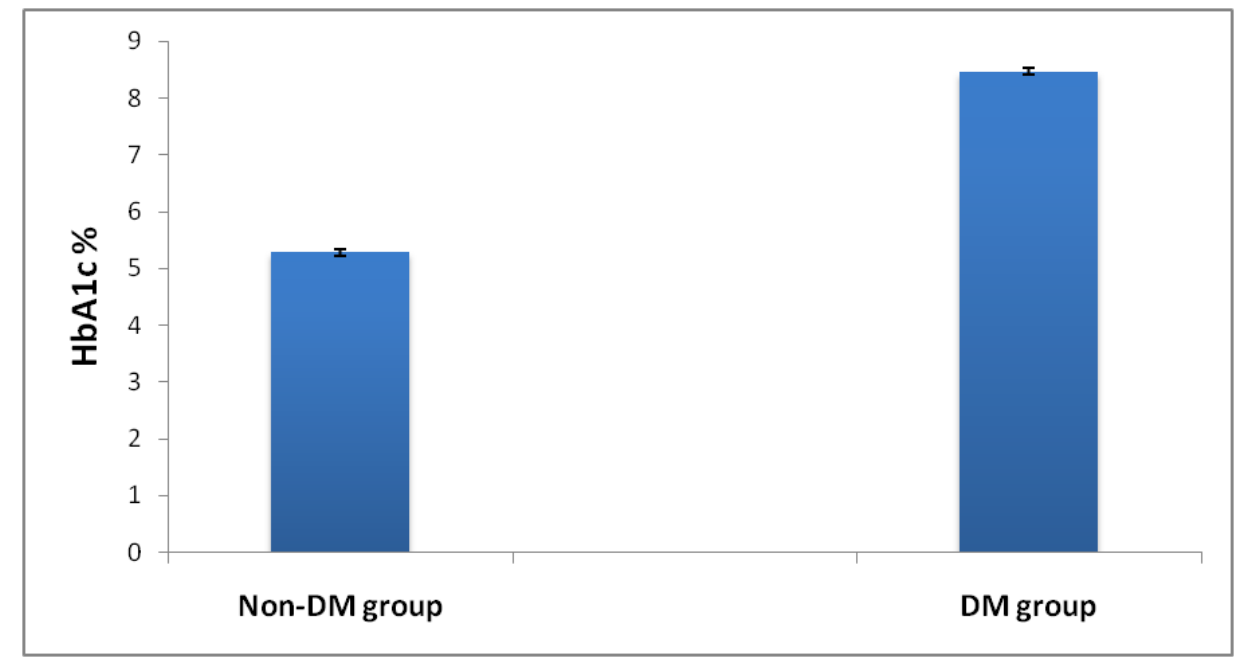

Figure 2: HbA1c parameters in diabetic and non-diabetic groups. 
In this study, to obtain an accurate result, the number of control group should have been increased in order to approach the real prevalence in the Kurdish community. Many investigations claim any correlations between $\mathrm{H}$. pylori and DM. One of the studies was carried out in South-East of Iran from August to December 2011. They pointed out that no significant differences were observed between DM and $\mathrm{H}$. pylori infection. ${ }^{5}$ However, many studies are revealing having such correlations. One of the studies was conducted in the United Arab Emirates in June 2002 until August 2003. They took 210 patients with type II DM and 210 subjects with DM. Their results confirmed that there was a significant association between T2DM and $\mathrm{H}$. pylori infection. ${ }^{14}$ The point of this explanation is that in type II DM population, particularly in obese type II DM, are prone to possess more infection compared to their counterpart without DM. Additional supportive data have come from groups in the Netherlands, Italy, Turkey, and Africa. Recently, a meta-analysis conducted by Zhou et al. ${ }^{4}$ involved 14080 patients from 41 studies with a total $\mathrm{H}$. pylori infection rate of $42.29 \%$. The odds ratio $(\mathrm{OR})$ for $H$. pylori infection was increased to 1.33 among patients with diabetes, especially in patients with T2DM $(\mathrm{OR}=1.76)$. The first demonstration is that $\mathrm{H}$. pylori infection leads to an increased incidence of diabetes was in a study by Jeon et al. ${ }^{8}$ using a prospective cohort of 782 Latino individuals $>60$ years of age. A study was performed by Chen and Blaser $^{15}$, whom they have provided new insight into the association between the seroprevalence of $\mathrm{H}$. pylori infection and the mean levels of HbA1c in two large national surveys: the National Health and Nutrition Examination Survey (NHANES) III and the NHANES 1999-2000. Their report showed that $\mathrm{H}$. pylori seropositivity, and $\mathrm{H}$. pylori cag positivity, in particular, was associated with higher mean $\mathrm{HbA1c}$ levels, an association that persisted after excluding individuals with a history of diabetes mellitus and controlling for potential confounders. The association was evident mainly in adults over 18 years of age. They also showed a synergistic effect of $\mathrm{H}$. pylori and $\mathrm{BMI}$ on increased levels of $\mathrm{HbA1c}$, indicating the role of $\mathrm{H}$. pylori in impaired glucose tolerance in adults that may be potentiated by a higher BMI level. They confirmed that $H$. pylori have a statistically significant association with non-insulin dependent diabetes mellitus. ${ }^{1}$ Accumulated research is examining an association between $\mathrm{H}$. pylori and type II DM. Some of them showed the correlation between $\mathrm{H}$. pylori and T2DM. One of the main points connected with both of them is obesity. Metabolic disorder and obesity have related to type II DM and $\mathrm{H}$. pylori separately as they are prone to have infection and insulin resistance. These studies showed this association between $\mathrm{H}$. pylori and type II DM, and their subjects in the DM group are with higher BMI. This is means that obese or overweight subjects with type II DM are more likely to suffer $\mathrm{H}$. pylori rather than just DM.

\section{Conclusion}

It can be concluded that a significant difference in the rate of $\mathrm{H}$. pylori infection was observed in type II DM group compared to that in the non-DM group in this study. The number of positive $\mathrm{H}$. pylori does not only increase with the higher HbA1c parameters but also with higher BMI. Future studies should include more subjects in both DM and non-DM groups in order to be accurately representative of the population in Erbil city.

\section{Competing interests}

The authors declare that they have no competing interests.

\section{References}

1. He C, Yang Z, Lu NH. Helicobacter pylori infection and diabetes: is it a myth or fact? World J Gastroenterol 2014; 20(16):4607-17.

2. Du GL, Su YX, Yao H, Zhu J, Ma Q, Tuerdi A, et al. Metabolic risk factors of type 2 diabetes mellitus and correlated glycemic control/ complications: A cross-sectional study between 
rural and urban Uygur residents in Xinjiang Uygur autonomous region. PLoS One 2016; 11(9):e0162611.

3. Kitabchi AE1, Umpierrez GE, Miles JM, Fisher JN. Hyperglycemic crises in adult patients with diabetes. Diabetes Care 2009; 32(7):1335-43.

4. Zhou X, Liu W, Gu M, Zhou H, Zhang G. Helicobacter pylori infection causes hepatic insulin resistance by the c-Jun/miR-203/SOCS3 signaling pathway. J Gastroenterol 2015; 50(10):1027-40.

5. Jafarzadeh A1, Rezayati MT, Nemati M. Helicobacter pylori seropositivity in patients with type 2 diabetes mellitus in south-east of Iran. Acta Med Iran 2013; 51(12):892-6.

6. Simon L, Tornóczky J, Tóth $M$, Jámbor $M$, Sudár Z. The significance of Campylobacter pylori infection in gastroenterologic and diabetic practice. Orv Hetil 1989; 130(25):1325-9.

7. Borody T1, Ren Z, Pang G, Clancy R. Impaired host immunity contributes to Helicobacter pylori eradication failure. Am J Gastroenterol 2002; 97(12):3032-7.

8. Jeon $C Y$, Haan MN, Cheng C, Clayton ER, Mayeda ER, Miller JW, et al. Helicobacter pylori infection is associated with an increased rate of diabetes. Diabetes Care 2012; 35(3):520-5.

9. de Luis DA, de la Calle H, Roy G, de Argila CM, Valdezate S, Canton R, et al. Helicobacter pylori infection and insulin-dependent diabetes mellitus. Diabetes Res Clin Pract 1998; 39(2):143-6.

10. Gentile S, Turco S, Oliviero B, Torella R. The role of autonomic neuropathy as a risk factor of Helicobacter pylori infection in dyspeptic patients with type 2 diabetes mellitus. Diabetes Res Clin Pract 1998; 42(1):41-8.

11. Bener A, Micallef R, Afifi M, Derbala M, Al-Mulla HM, Usmani MA. Association between type 2 diabetes mellitus and Helicobacter pylori infection. Turk J Gastroenterol 2007; 18:225-9.

12. Devrajani BR, Shah SZ, Soomro AA, Devrajani T. Type 2 diabetes mellitus: A risk factor for Helicobacter pylori infection: A hospital based case-control study. Int $\mathrm{J}$ Diabetes Dev Ctries 2010; 30(1):22-6.

13. Ko GT, Chan FK, Chan WB, Sung JJ, Tsoi C, To $\mathrm{K}$, et al. Helicobacter pylori infection in Chinese subjects with type 2 diabetes. Endocrine Research 2001; 27:171-7.

14. Bener A, Micallef R, Afifi M, Derbala M, Al-Mulla HM, Usmani MA. Association between type2 diabetes mellitus and Helicobacter pylori infection. Turk J Gastroenterol 2007;18(4):225-9.

15. Chen $\mathrm{Y}$, Blaser MJ. Association between gastric Helicobacter pylori colonization and glycated hemoglobin levels. J Infect Dis 2012;205(8):1195 -202 . 